\title{
Editorial
}

\section{Functional Nanomaterials for Energy Conversion and Storage}

\author{
Tianyi Kou, ${ }^{1}$ Gongming Wang, ${ }^{2}$ Xihong Lu, ${ }^{3}$ Yang Song, ${ }^{4}$ and Teng Zhai ${ }^{5}$ \\ ${ }^{1}$ University of California, Santa Cruz, CA 95064, USA \\ ${ }^{2}$ University of California, Los Angeles, CA 90095, USA \\ ${ }^{3}$ Sun Yat-sen University, Guangzhou 510275, China \\ ${ }^{4}$ Oak Ridge National Laboratory, Oak Ridge, TN 37831, USA \\ ${ }^{5}$ Nanjing University of Science and Technology, Nanjing 210094, China \\ Correspondence should be addressed to Teng Zhai; tengzhai@njust.edu.cn
}

Received 26 July 2016; Accepted 26 July 2016

Copyright (C) 2016 Tianyi Kou et al. This is an open access article distributed under the Creative Commons Attribution License, which permits unrestricted use, distribution, and reproduction in any medium, provided the original work is properly cited.

Fossil fuels have been the primary energy source in the past thousands of years. However, the nonrenewable feature and air pollutants produced by fossil fuel combustion make fossil fuels unfavorable for long-term usage, under the background of global population booming. In order to relieve this issue, it is urgent to develop renewable energy storage/conversion devices with both high energy and power delivering performances. A variety of energy storage/conversion devices have been developed such as lithium ion batteries and supercapacitors. Lithium ion batteries usually deliver higher energy density around $200 \mathrm{Wh} / \mathrm{kg}$ because of the bulk intercalation of a large amount of lithium ions. However, resulting from the sluggish bulk faradaic reactions, a high degree of bulk intercalation of lithium ions should be guaranteed by the lower charging and discharging rate. This fact leads to the inferior power delivering ability of lithium ion batteries with typical power density on the level below $1 \mathrm{~kW} / \mathrm{kg}$. To supplement the drawbacks of power delivering in lithium ion batteries, supercapacitors have been therefore put forward to aim at high rate charging and discharging. Due to the fast interface charge storage mechanism, supercapacitors can provide power density on the level of tens of $\mathrm{kW} / \mathrm{kg}$, much higher than that in lithium ion batteries. Unfortunately, the fast interfacial charge storage mechanism in supercapacitors is contradictory to sluggish bulk charging mechanism which results in lower energy density for supercapacitors and several $\mathrm{Wh} / \mathrm{kg}$ are usually seen.

In the scenario of both intrinsic problems existent in lithium ion batteries and supercapacitors, a balance between energy and power should be required so that energy density is boosted and at the same time decent power density is also kept. A key method to resolve this long-standing problem should reside in developing novel nanostructured electrodes for these electrochemical energy storage devices. High conductivity, fast ions intercalation channel, highly electrolyte ions accessible surface, rich faradaic reactions sites, and stable long duration charging/discharging performance are indispensable to high efficient lithium ion batteries or supercapacitors. And these are also the common goals for materials scientists when developing state-of-the-art electrodes. Based on these goals, this special issue includes reports selected by the guest editors team which are focused on the advanced nanomaterials synthesis or/and the application of nanostructured electrodes in energy storage/conversion devices such as lithium ion batteries and supercapacitors. We hope the work summarized in the issue can provide some new ideas for our science community and inspire our colleagues studying functional materials for energy storage/conversion.

\section{Acknowledgments}

The guest editors team sincerely appreciates the contribution and efforts from all of the authors and referees to make this special issue a success.

Tianyi Kou

Gongming Wang

Xihong Lu

Yang Song

Teng Zhai 

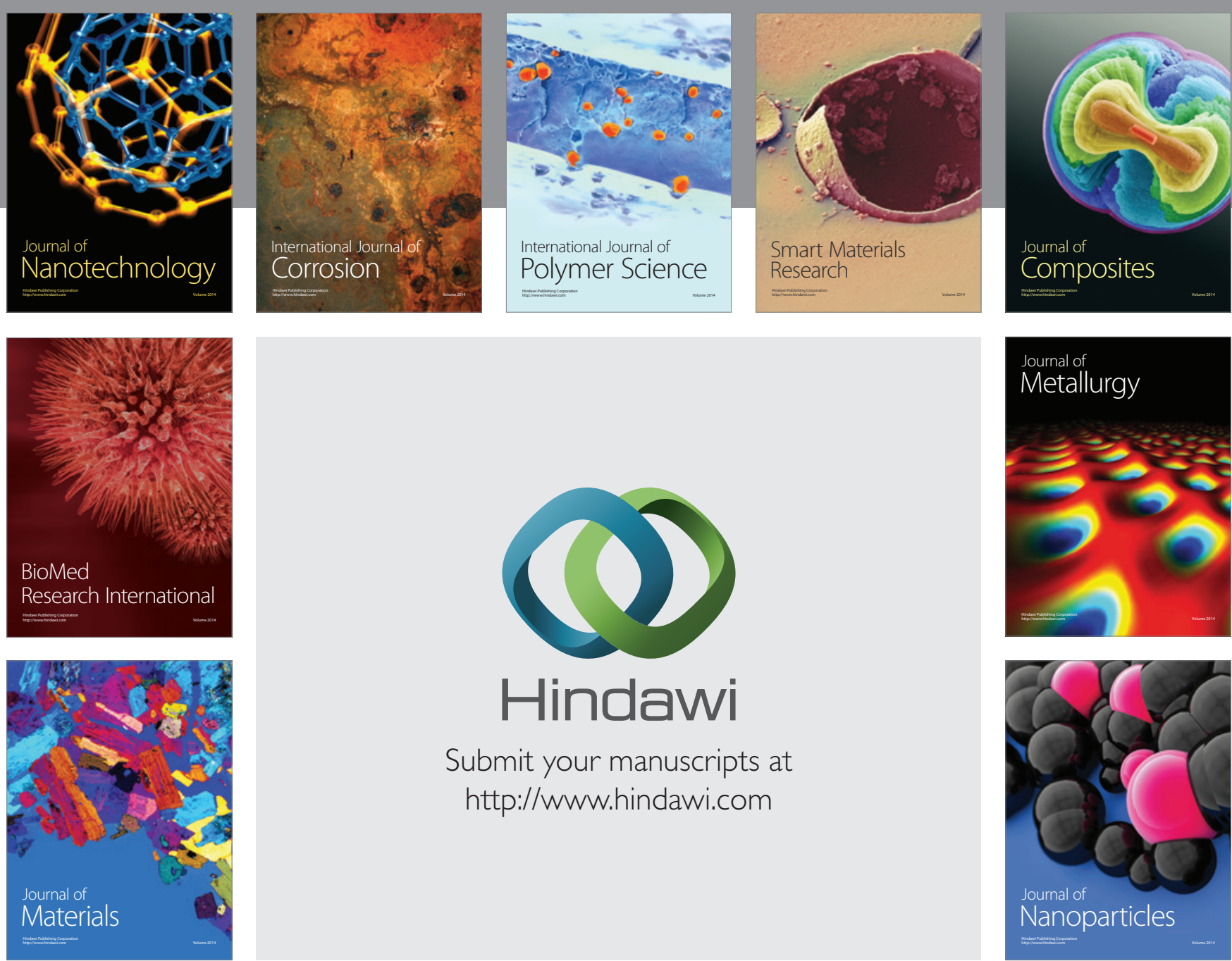

\section{Hindawi}

Submit your manuscripts at

http://www.hindawi.com

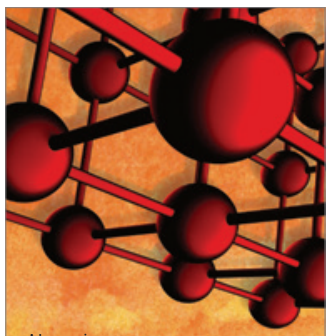

Materials Science and Engineering
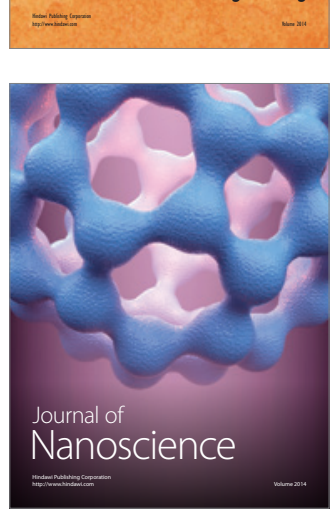
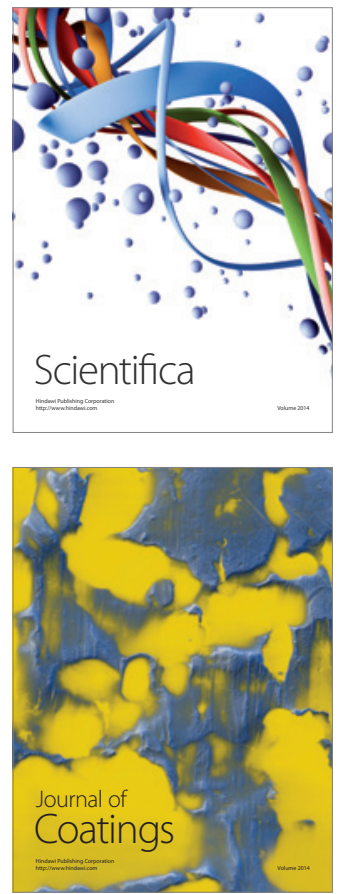
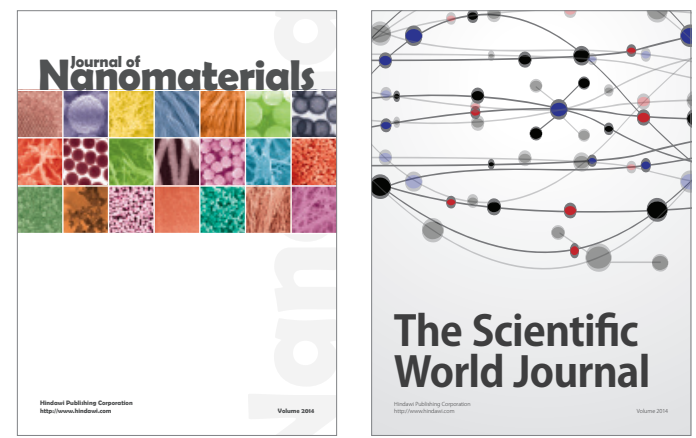

The Scientific World Journal
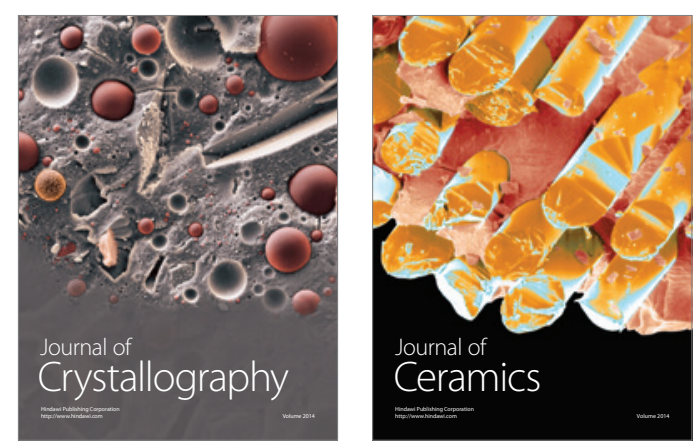
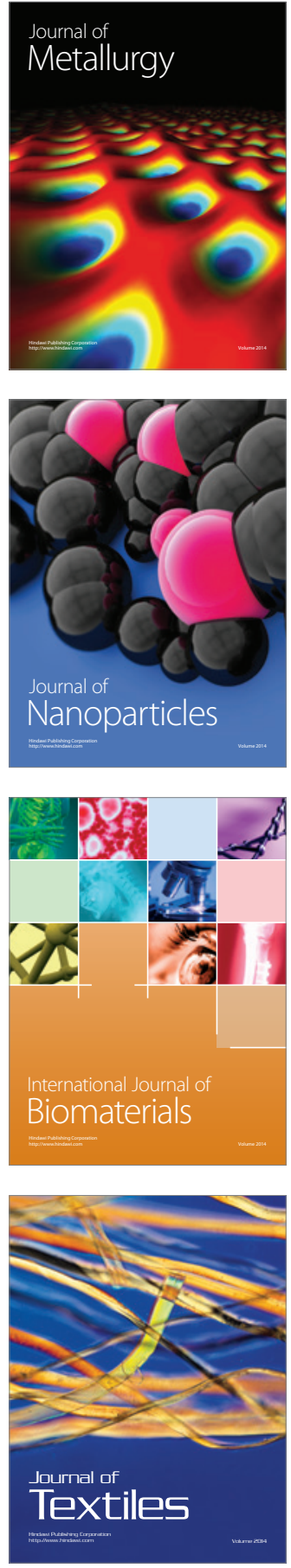